\title{
Zytologische Untersuchungen über die Ganglienzellen der Kaninchenzunge.
}

\author{
Von \\ Hiroşhi Suzuki und Hiroshi Ookubo. \\ Aus dem Anatomischen Institut der Hochschule für \\ Zahnheilkunde, Nippon Universität zu Tokyo.
}

Mit 8 Figuren in Tafel XV.

Inhalt.

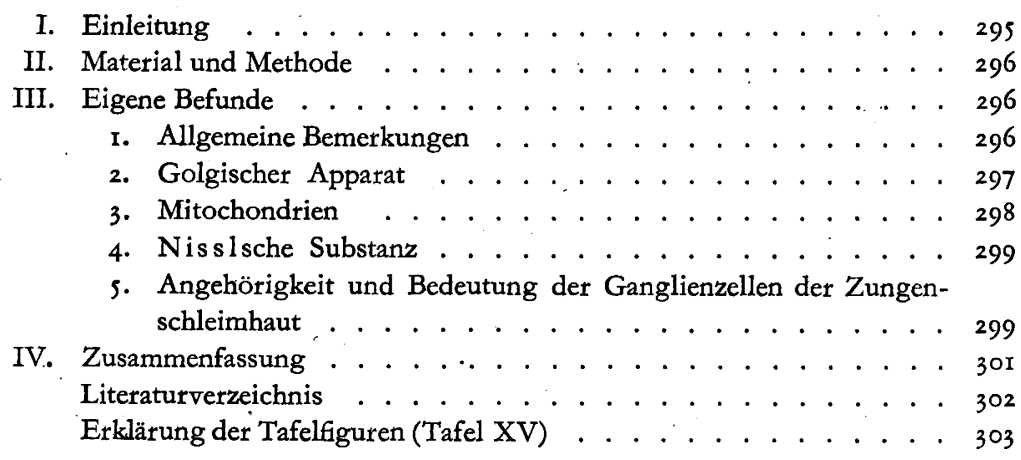

\section{Einleitung.}

Die Tatsache, daß die Schleimhaut der Zunge in bestimmten Stellen die Gangliepzellen enthält, ist von alters an bekannt. Doch fehlen bis jetzt die zytologischen Kenntnisse über diese Ganglienzellen nahezu gänzlich. Somit haben wir in der vorliegenden Untersuchung an der Kaninchenzunge die Ganglienzellen der Zungenschleimhaut zytologisch von verschiedenen Seiten erforscht, um die Beschaffenheit des Golgiapparates, der Mitochondrien und der Nisslsubstanz kennenzulernen.

Die Frage der Angehörigkeit der Ganglienzellen der Zungenschleimhaut zu dem zerebrospinalen Nervensystem oder zu dem vegetativen Nervensystem steht heute noch offen. Meiner Ansicht nach ist die Kenntnis der feineren Zytoplasmastrukturen dieser Ganglienzellen zur Lösung der betref- 
fenden. Frage unentbehrlich. Neuerdings wurden die Eigenschaften des Golgiapparates, der Nisslschen Substanz u.a. der peripheren vegetativen Ganglienzellen durch die zytologischen Untersuchungen von I to (1936), Ito, und Nagahiro (1937), Ito und Aoki (1939) und Ito und Kubo (1940) klargestellt. Auf Grund der Ergebnisse dieser Autoren kam Hioki (194I) in seiner Untersuchung über die Ganglienzellen in der Gegend der Papilla vallata der menschlichen Zunge zum Schlusse, daß die Ganglienzellen in der Zungenschleimhaut dem vegetativen Nervensystem augehören sollen. Wir haben in dieser Untersuchung die Ganglienzellen in der Gegend der Papilla foliata der Kaninchenzunge studiert.

\section{Material und Methode.}

Die Gewebestücke, welche Papilla foliata enthalten, wurden aus dem Margo lateralis der Zunge von gesunden Kaninchen herausgeschnitten und nach dem Zwecke mit den folgenden Fixierungsverfahren behandelt.

Für die Darstellung des Golgiapparates haben wir die Kolatche vsche Methode angewandt; die mit $\mathrm{Ch}$ a mp y scher Flüssigkeit 2 Tage lang fixierten Gewebestücke kamen nach der Waschung mit fliessendem Wasser in eine $1 \%$ ige Osmiumsäurelösung, darin wurden sie bei 3s C 6-7 Tage lang osmiert. Für den Nachweis der Mitochondrien wurden die Gewebestücke mit der Ch a m p y schen Flüßigkeit 2 Tage lang fixiert, darauf wurden sie in $3 \%$ iger Kaliumbichromatlösung, Tage lang nachchromiert. Die Schnitte wurden mit Eisenhämatoxylin nach Heid en ha in gefärbt. Die Levi sche Fixierungsmethode wurde auch benutzt. Für die Darstellung der Nisslschen Substanz wurden die mit Zenker-Formol fixierten Schnitte nach Spiel meyer mit Toluidinblau gefürbt.

Die fixierten Gewebestücke kamen alle nach der Entwässerung durch aufsteigender Alkoholreihe in Xylol und wurden dann in Paraffin eingebettet und darauf in $4-5 \mu$ dicke Serienschnitte zerlegt.

\section{Eigene Befunde.}

\section{Allgemeine Bemerkungen.}

Die Verteilung der Ganglienzellen in der Zungenschleimbaut wurde von Kölliker (1899), Takakusu (1924), Kolmer (1927), Hioki (1941) u.a. berichtet. Nach diesen Autoren kommen, die Ganglienzellen hauptsächlich in der Tela submucosa vor, und zwar in der Gegend der Zungenwurzel, insbesondere der Papilla vallata und der Papilla foliata; sie sind in übrigen Stellen kaum zu finden. Wir haben in der vorliegenden Untersuchung in 
dem lateralen Rand der Kaninchenzunge die Ganglienzellen überhaupt in der Tela submucosa unmittelbar unterhalb der Papilla foliata vorgefunden. Sie kommen außerdem noch häufig innerhalb der Zungendrüse zwischen den Hàuptstücken vor. Gewöhnlich bilden sie kleine Anhäufungen, welche mitunter im Laufe der Nervenfaserbündel eingeschaltet sind. Die Lamina propria mucosae führen nur äußerst selten verstreut die Ganglienzellen. Hioki (I94I) hat in einem Falle der menschlichen Zunge die Anhäufung der Ganglienzellen innerhalb der Papilla vallata gefunden. Aber wir haben in dieser Untersuchung innerhalb der Lamina propria der Papillae foliatae niemals die Ganglienzellen bemerkt.

Die Größe der Ganglienzellen variiert in hohem Maße; nach der Größe können wir sie in große, mittelgroße und kleine Ganglienzellen einteilen. Durch die Untersuchungsmethoden, welche wir hier angewandt haben, werden die Fortsätze der Ganglienzellen nicht dargestellt, so erscheinen die Ganglienzellen lediglich rundlich, oval und abgerundet vieleckig gestaltet.

Der Kern ist groß und rundlich bis elliptisch geformt und sieht immer hell aus. In dem Kernraum finden sich rundliche Kernkörperchen ein- bis mehrfach.

Die Lage des Kerns im Zytoplasma ist sehr wichtig. Ito (1936), Ito und $\mathrm{Kubo}$ (1940) und Hioki (194I) haben alle bei der zytologischen Untersuchung der peripheren vegetativen Ganglienzellen darauf aufmerksam gemacht, daß bei diesen vegetativen Ganglienzellen der Kern immer stark exzentrisch gelagert ist. Diese Tatsache haben sie als eine wichtige Eigenschaft der betreffenden Zellen vorgeschlagen. Wir haben bei den Ganglienzellen in der Gegend der Papilla foliata der Kaninchenzunge die Befunde der genannten Forscher bestätigt. Wie in den Figuren gezeichnet, liegt der Kern der Ganglienzellen von Kaninchenzunge immer stark exzentrisch, so $\mathrm{da} ß$ der Kern in einer Seite dem reichlichen Zytoplasma zukehrt, aber in der' entgegengesetzten Seite von einer dünnen Zytoplasmaschicht umgeben ist.

Hioki (194I) hat in den Ganglienzellen der menschlichen Zunge die Pigmentgranula wahrgenommen, doch haben wir in denen der Kaninchenzunge diese nicht gefunden.

\section{Golgiapparat.}

Der Golgiapparat der Ganglienzellen von Kaninchenzunge besteht aus den mehr oder weniger gewundenen, durch Osmiumimprägnation schwarz angefärbten Strängen. Diese Stränge bilden sich miteinander verbindend ein Netzwerk (Fig. 1-3). Der Golgiapparat liegt immer auf der Kernmembran, aber bei keinem-Falle um gibt der Netzapparat den Kern von allen Seiten kon- 
zentrisch. In dieser Hinsicht weicht der Golgiapparat der Ganglienzellen in Zungenschleimhaut von demselben der Ganglienzellen der zerebrospinalen Nervensystems, insbesondere der Spinalganglienzellen, deutlich ab. Hier lagert sich der Golgiapparat immer auf einer Seite des Kerns lokalisiert, wo das Zytoplasma der Ganglienzellen am reichlichsten zu finden ist. Ito (1936), Ito und Nagahiro (1937) und Ito und A oki (1939) haben bei den Ganglienzellen in der Darmwand und in der Gl. submaxillaris des Hundes auf die gleiche besondere Verteilung des Golgiapparates im Zytoplasma aufmerksam gemacht und faßten dies als eine wichtige morphologische Eigenschaft der peripheren vegetativen Ganglienzellen auf. Hioki hat neuerdings bei den Ganglienzellen der menschlichen Zunge das Vorhandensein der gleichen Verteilung des Golgiapparates von der Verteilung der Saftkanälchen (GolgiNegativ) vorgestellt.

Wie oben erwähnt, behält der Golgiapparat mit dem Kern eine innige Beziehung; er liegt immer unmittelbar der Kernmembran an und sein Netzwerk dehnt von dort an nach der Peripherie aus. Bei der einen Anzahl der Ganglienzellen ist die Ausdehmung hochgradig und das Netzwerk erstreckt sich bis zur Randzone des Zytoplasma (Fig. I u. 2), bei der anderen ist der Golgiapparat in der den Kern unmittelbar umgebenden Plasmazone beschränkt und bildet auf der Kernmembran ein gut umschriebenes Netzwerk (Fig. 3). Das gleiche Verhältnis haben I to und Nagahiro (1937) bei den Ganglienzellen des Plexus myentericus und submucosus des Rattendarmes bemerkt. Nach ihren Befunden dehnt der Golgiapparat der Ganglienzellen des Plexus myentericus weit nach der Peripherie aus und derselbe der Ganglienzellen des Plexus submucosus bildet ein auf der Kernmembran beschränktes, gut umschriebenes Netzwerk.

\section{Mitochondrien.}

Das Chondriom der vegetativen Ganglienzellen haben I to (1936) und Ito und Nagahiro (1937) und Ito und Aoki (1939) u.a. beschrieben. Nach I to und I to und Nag a h i ro sind die Mitochondrien der intramuralen Ganglienzellen der Darmwand faden-, stäbchenförmig und granulär gesstaltet und verteilen sich im ganzen Zytoplasma nahezu gleichmäßig. It o und Aoki haken in den Ganglienzellen in der Gl. submaxillaris des Hundes nur granuläre Mitochondrien wahrgenommen.

Nach unseren eigenen Beobachtongen sind die Mitochondrien der Ganglienzellen der Kaninchenzunge vorwiegend faden-und stäbchen förmig und mehr oder weniger geschlängelt im ganzen Zytoplasma gleichmäßig verteilt. Die granulären Mitochondrien sind im allgemeinen in sehr kleiner Zahl zu finden (Fig. 4, 5). 


\section{Nis s lsche Substanz. :}

Die Nisslsche Substanz der Ganglienzellen' der Kaninchenzunge stellt sehr feine Granula dar, welche im ganzen Zytoplasma diffus verteilt sind und nie die typischen, scharf begrenzten Tigroidschollen bilden, wie von Ito und anderen genannten Forschern bei den peripheren vegetativen Ganglienzellen berichtet wurde. Die Granula sammeln sich aber am häufigsten in der Randzone des Zytoplasma zu einer dichten Anhäufung und bilden sogenannten ,peripheren Ring“. Bald bildet dieser periphere Ring einen kontinuietlichen Ring (Fig. 6), bald ist er stellenweise unterbrochen (Fig. 7) und bald ist er in einer kürzeren Strecke der Randzone beschränkt (Fig. 8). Der ,perinukleäre Ring“", welcher von Ito und Ito und Kubo in den Ganglienzellen der Darmwand beschrieben wurde, kommt in den Ganglienzellen der Kaninchenzunge kaum vor. Außerdem haben wir selten solche Ganglienzellen bemerkt, in welchen die Nisslsche Granula weder den peripheren noch den perinukleären Ring bilden und im ganzen Zytoplasma fast gleichmäßig verteilt sind.

I to und $\mathrm{Ku}$ bo haben in der zytologischen Untersuchung der intramuralen Ganglienzellen des menschlichen Darmes bemerkt, daß das Zytoplasma der Ganglienzellen des Plexus submucosus bei der Toluidinblaufärbung viel dunkler angefärbt wird als das der Ganglienzellen des Plexus myentericus. Nach unserer Untersuchung färbt sich das Zytoplasma der Ganglienzellen der Kaninchenzunge durch die Toluidinblaufärbung im allgemeinen dunkel an, so stimmen sie in dieser Hinsicht mit den Ganglienzellen des Plexus submucosus des Darmes überein.

Ito (1936) und Ito und Nagahi ro (1937) und Ito und $\mathrm{Kub}$ o (1940) haben die Nisslsche Substanz der intramuralen vegetativen Ganglienzellen der Darmes untersucht und sind zu der Kenntnis gekommen, daß die Niss lsche Substanz in diesen Ganglienzellen diffus im Zytoplasma verteilt ist, ohne die typischen Tigroidschollen zu bilden, und $\mathrm{da} ß$ sie aber häufig in der peripheren Zone oder in der perinukleären Zone des Zytoplasma sich dicht ansammelt und dort den "peripheren" und ,petinukleären Ring“ bildet. Sie haben diese besondere Verteilung der Nisslschen Substanz als eine wichtige, zytologische Eigenschaft der peripheren vegetativen Ganglienzellen angesehen. Unsere Ergebnisse bei den Ganglienzellen der Zungenschleimhaut stimmen im großen ganzen mit denjenigen der eben genannten Autoren überein.

\section{Angehörigkeit und Bedeutung der Ganglienzellen der Zungenschleimhaut.}

Die Frage über die Angehörigkeit der Ganglienzellen in der Zungen- 
schleimhaut $z \mathfrak{u}$ dem zerebrospinalen Nervensystem oder $z \mathfrak{u}$ dem vegetativen ist heute noch nicht endgültig beantwortet. Lenhossék (1894) ist der Meinung, daß die Nervenzellen in der Gegend der Papilla vallata wenigstens zu Teil zum Sympathicus gehören sollen. Kolmer (1927) schließt der Lenhossék's Meinung an, doch hat ex anderseits erwähnt, daß es schwierig ist, die Frage zu entscheiden, ob die Nervenzellen in der Gegend der Papilla vallata die bipolaren Nervenzellen im peripheren Teil des N. glossopharyngeus sind oder nicht. Nach Stew atd (1920) stammen die Ganglienzellen im hinteren Drittel der Funge von N. glossopharyngeus ab und nach seiner Ansicht dürfte dies auch der Fall bei den Ganglienzellen sein, welche unterhalb der Geschmacksknospen vorkommen. Takakusu (1924) hat vorgenommen, die Angehörigkeit der Ganglienzellen der Zungenschleimhaut durch die bei der Adrenalin und Pilocarpin-Injektion hervorgerufene Veränderung der Nisslscken Substanz zu entscheiden und kam zum Schlusse, daß die Ganglienzellen in Zungenwurzel dem Parasympathius angehören und die kleinen im übrigen Zungenabschnitten wahrscheinlich genetisch mit den Spinalganglienzellen identisch sein sollen.

Neuerding hat Hioki (194I) in seiner Untersuchung der Ganglienzellen in der Gegend der Papilla vallata geschlossen, daß die Ganglienzellen der Zungenschleimhaut dem vegetativen Nerversystem gehören sollen, weil sie in der stark exzentrischen Lage des Kerns und in der speziellen Lokalisation der Saftkanälchen (Golgi-Negativ) mit den von Ito und anderen Forschem untersuchten vegetativen Ganglienzellen zusammenfallen.

Nach unserer eigenen zytologischen Untersuchung stimmen die Ganglienzellen in der Gegend der Papilla foliata der Kaninchenzunge, wie oben eingehend beschrieben, in der stark exzentrischen Lage des Kerns, der speziellen Lokalisation des Golgiapparates und in den Beschaffenheiten der Nis sschen Substanz mit den von I to und seinen Mitarbeitern untersuchten vegetativen Ganglienzellen vollkommen überein. So dürfen wir nun angeben, daß die Ganglienzellen in der Zungenschleimhaut dem vegetativen Nervensystem gehören. Wie oben erwähnt, stimmen die Ganglienzellen in der Gegend der Papilla foliata nach dem Befunde der Niss1 -Färbung mit Toluidinblau mit den des Plexus submucosus des Darmes übetein, und die Ganglienzellen des Plexus submucosus sollen, nach der experimentellen Untersuchung von Takakusu (1924) dem Parasympathicus angehören; daher entsteht die Annahme, daß die Ganglienzellen der Zungenschleimhaut wahrscheinlich dem Parasysmpathicus angehörig sein dürften.

Was die. Frage, über die Bedeutung der Ganglienzellen der Zungenschleimhaut angeht, so hat Kolmer darüber angenommen, daß die Ganglienzellen der Zungensschleimhaut in einer Beziehung mit der Zungendrüse 
stehen sollen. Hioki hat bei einer menschlichen Zunge das Vorkommen der Ganglienzellen innerhalb der Papilla vallata bemerkt und hat folgende Schlußfolgerung gezogen, daß die Ganglienzellen der Zungenschleimhaut nicht nur mit der Drüse, sondern auch mit der Funktion der Geschmacksknospen zu tun zu haben scheinen. Neuerdings hat Suzuki (1943), einer von uns, in seiner eingehenden zytologischen Untersuchung der Geschmacksknospen die wichtige Tatsache entdeckt, daß die Stützzellen der Geschmacksknospen höchstwahrscheinlich die sekretorische Tätigkeit besitzen, welche ansehnlich die Funktion der Geschmackszellen zu regulieren scheinen. Somit ist die Angabe von Hioki sehr interessant.

Wir haben in der vorliegenden Untersuchung festgestellt, daß die Ganglienzellen in der Gegend der Papilla foliata häufig in dem interstitiellen Bindegewebe der Zungendrüse auftreten, und daß sie zum vegetativen Nervensystem gehören, so unterliegt keinem Zweifel, daß sie mit der Funktion der Zungendrüse zu tun haben. Nach unserer Beobachtung kommen sie niemals innerhalb der Papilla foliata vor, doch scheint uns die Annahme auch möglich zu sein, daß sie mit den Geschmacksknospen der Papilla foliata in einer Beziehung ștehen dürften, wie von $\mathrm{Hioki}$ bei der Papjila vallata angenommen wurde.

\section{Zusammenfassung.}

Die wichtigen Ergebnisse dieser zytologischen Untersuchung der GangJienzellen in der Gegend der Papilla foliata der Kaninchenzunge werden im folgenden zusammenfassend angefür $\mathrm{rt}$.

x. In dem lateralen Rand der Kaninchenzunge sind die Ganglienzellen hauptsächlich in der Gegend der Papilla foliata und zwar vorwiegend in der Tela submucosa und im interstitiellen Bindegewebe der Zungendrüse als keine Gruppen zu finden.

2. Die Größe der Ganglierzellen ist in hohem Maße variierend, aber ihr Kern ist immer stark exzentrisch vorhanden.

3. Ganglienzellen der Kaninchenzunge führen nicht Pigmentgranula.

4. Der Golgiapparat stellt ein Netzwerk dar und liegt auf einem, dem reichlichen Zytoplasma zugekehrten Kernpol; deshalb um gibt das Netzwerk, -wie bei den Spinalganglienzellen, nicht den Kern konzentrisch.

s. Die Mitochondrien sind stäbchen-und fadenförmig gestaltet und im ganzen Zytoplasma annähernd gleichmäßig verteilt.

6. Die Nis slsche Substanz verteilt sich im ganzen Zytoplasma diffus und bildet keine Tigroidschollen. Sie sammelt sich aber sehr häufig in der Randzone des Zelleibes besonders dicht an, um den sogenannten „peri- 
pheren Ring “' zu bildèn.

7. In der oben angeführten stark exzentrischen Lage des Kerns, der besonderen Anordnung des Golgiapparates und der Beschaffenheit der N is s 1 schen Substanz stimmen die Ganglienzellen der Zunge mit den von Ito und seinen Mitarbeitern untersuchten, peripheren, vegetativen Ganglienzellen gut überein, so kamen wir zum Schlusse, daß die Ganglienzellen der Zungenschleimhaut, wie von $\mathrm{Hioki}$ neuerdings angegeben, dem vegetativen Nervensystem angehören. Sie scheinen wahrscheinlich mit der Tätigkeit der Zungendrüse und der Geschmacksknospen in einer Beziehung zu stehen.

Zum Schlusse sprechen wir Herrn Prof. Dr. T. Taniguchi für seine stete Unterstützung und Herrn Dr. T. It o für seine freundliche Leitung bei Ausführung dieser Arbeit unseren herzlichsten Dank aus.

\section{Literaturverzeichnis.}

I) Ito, T., 1936. Zytologische Untersuchungen über die intramuralen Ganglienzellen des Verdauungstraktes. Uber die Ganglienzellen der menschlichen Wurmfortsätze etc. Okajimas Fol. anat. jap. Bd. 14. S. 621.

2) Ito, T., und K. Nagah i ro, 1937. Zytologische Untersuchungen über die intramuralen Ganglienzellen des Verdauungstraktes. Uber die Ganglienzellen der Darmwand der Ratte etc. Okajima Fol. anat. jap. Bd. 15. S. 609.

3) I to, T. und S. A oki, 1939. Uber den Golgiapparat der Ganglienzellen der Gl. submaxillaris des Hundes. Okajimas Fol. anat. jap. Bd. 17. S. 567.

4) Ito, T. und M. Kubo, 1940: Zytologische Untersuchungen über die intramuralen Ganglienzellen des menschlichen Darmes mit besonderer Berücksichtigung auf die Nisslsche Substanz. Cytologia. Bd. ro. S. 334.

5) Hioki, K., r94x. Uber das Vorkommen der Nervenzellen in dex Papilla vallata der menschlichen Zunge. Mitteilungen aus der japanischen orologischen Gesellschaft. Bd. 34 . S. 305. (Japanisch).

6) Kölliker, r899. Gewebelehre. Leipzig. (zit. nach Takakusu).

7) Kolmer, W., 1927. Geschmacksorgan. Möllendorffs Hand b. d. mikr. Anat. d. Mensch. Bd. I/III. S. 154 .

8) Lenhossek, 1894. Beiträge zur Histologie des Nervensystems und der Sinnesorgane. (zit. nach Kolmer).

9) Steward, r920. On the origin of the ganglion cells of the nervus terminalis of the albino rat. Journ. comp. Neurol: Vol. 32. p. 99. (zit. nach Kolmer).

-10) Suzuki, H., i943. Zytologische Untersuchungen über die Geschmacksknospe der Kaninchenzunge mit besonderer Berücksichtigung der Sekretion ähnlichen Erscheinung der Stützzellen. Okajimas Fol. anat. jap. Bd. 22 S. 1943.

II). Takakusu, R., x923a. Úber die Veränderung der Nervenzellen durch Adrenalin und Pilocarpininjektion. Nissin-Igaku. Bd. 14. S. 71. (Japanisch).

12) Takakusu R., 1923b. Uber die Angehörigkeit des Eingeweidenerven zum Sympathicus (Eine histologische Untersuchung). Nissin-Igaku. Bd. I4. S. I89. (Japanisch). 
Zytologische Untersuchungen über die Ganglienzellen der Kaninchenzunge.

Erklärung der Tafelfiguren (Tafel XV);

Alle Figuren wurden mit dem A bbeschen Zeichenapparat auf der Tischhöhe gezeichnet und zwar mit $Z$ e i $\beta$ Achromat-Imm., n.A. 1.25, Kompensationsokular 12. Alle Figuren wurden beim Abdruck auf $2 / 3$ verkleinert.

Fig. 1-3. Golgiapparat der Ganglienzellen in der Zungenschleimhaut. Kolatchevsche Osmiumimprägnationsmethode.

Fig. 4-5. Mitochondrien der Ganglienzellen in der Zungenschleimhaut. Cha m p ysche Fixierungsgemisch, Eisenhämatoxyline nach $\mathrm{Heidenhain.}$

Fig. 6-8. Niss 1 sche Substanz der Ganglienzellen in der Zungenschleimhaut. ZenikerFormol, Toluidinblaufärbung nach S pielmeyer. 

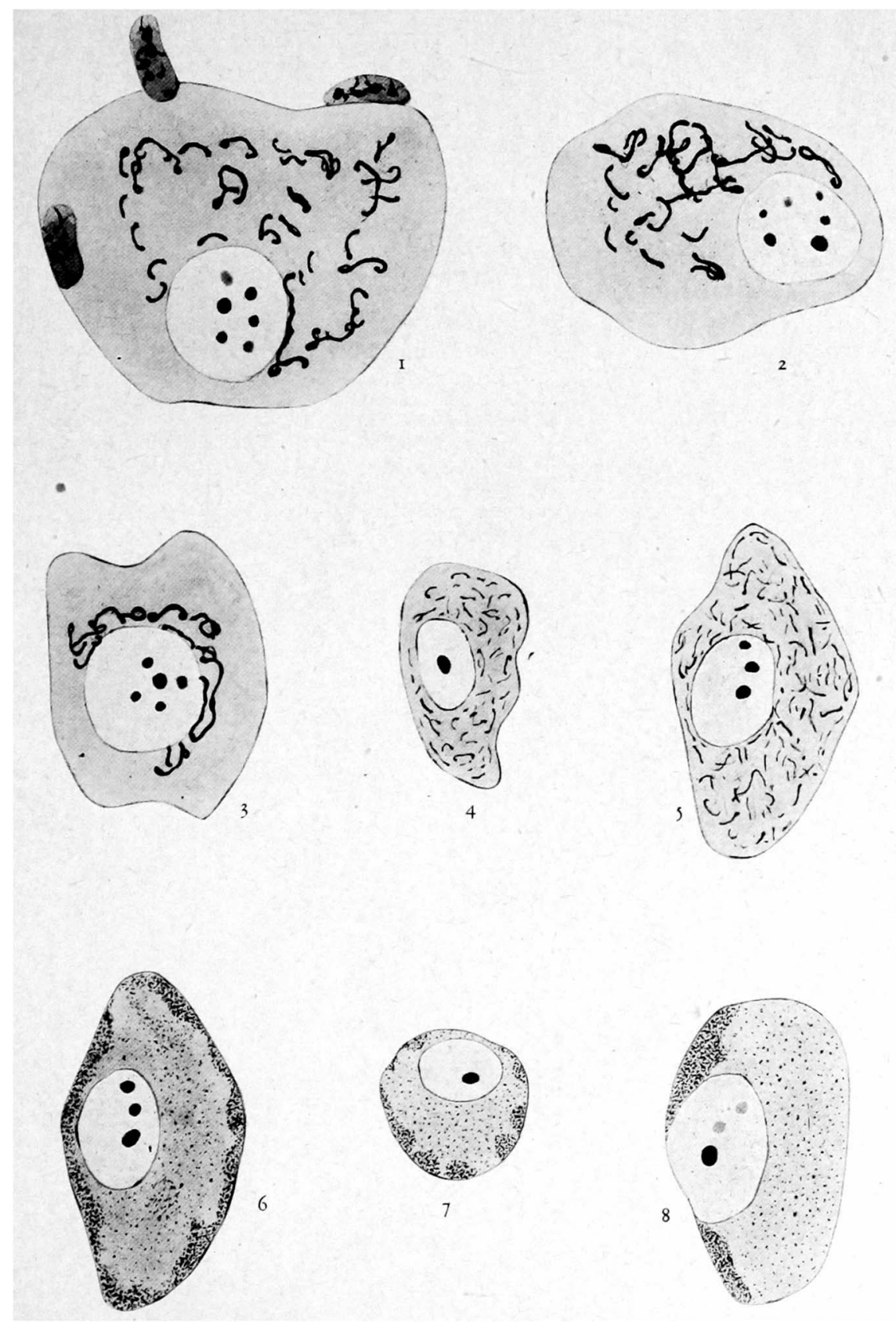

Suzuki u. Ookubo. 